\title{
L-Arginine Improves Endothelium-dependent Vasodilation in Hypercholesterolemic Humans
}

Mark A. Creager, Shelly J. Gallagher, Xavier J. Girerd, Sharon M. Coleman, Victor J. Dzau, and John P. Cooke

Vascular Medicine and Atherosclerosis Unit, Brigham and Women's Hospital and Harvard Medical School, Boston, Massachusetts 02115

\begin{abstract}
Endothelium-dependent vasodilation is impaired in hypercholesterolemia, even before the development of atherosclerosis. The purpose of this study was to determine whether infusion of L-arginine, the precursor of the endothelium-derived relaxing factor, nitric oxide, improves endothelium-dependent vasodilation in hypercholesterolemic humans. Vascular reactivity was measured in the forearm resistance vessels of 11 normal subjects ( serum LDL cholesterol $=2.76 \pm 0.10 \mathrm{mmol} /$ liter $)$ and 14 age-matched patients with hypercholesterolemia (serum LDL cholesterol $=4.65 \pm 0.36 \mathrm{mmol} /$ liter, $P<0.05)$. The vasodilative response to the endothelium-dependent vasodilator, methacholine chloride, was depressed in the hypercholesterolemic group, whereas endothelium-independent vasodilation, induced by nitroprusside, was similar in each group. Intravenous administration of $\mathrm{L}$-arginine augmented the forearm blood flow response to methacholine in the hypercholesterolemic individuals, but not in the normal subjects. $L$-arginine did not alter the effect of nitroprusside in either group. D-arginine had no effect on forearm vascular reactivity in either group. It is concluded that endothelium-dependent vasodilation is impaired in hypercholesterolemic humans. This abnormality can be improved acutely by administration of $\mathrm{L}$-arginine, possibly by increasing the synthesis of endothelium-derived relaxing factor. (J. Clin. Invest. 1992. 90:1248-1253.) Key words: L-arginine • cholesterol • endothelium • endothelium-derived relaxing factor $\bullet \operatorname{limb}$ blood flow
\end{abstract}

\section{Introduction}

The endothelium plays a pivotal role in maintaining homeostasis of the blood vessels. It synthesizes biologically active substances that regulate vascular tone, modulate blood cell-vessel wall interaction, prevent thrombosis, and influence smooth muscle cell growth ( 1 ). Important among these vasoactive substances is endothelium-derived relaxing factor (EDRF), ${ }^{1}$

Dr. Girerd's present address is Hopital Broussais, Paris, France; Dr. Dzau's and Dr. Cooke's present address is Falk Cardiovascular Research Center, Stanford University School of Medicine, Palo Alto, CA.

Address correspondence to Mark A. Creager, M.D., Cardiovascular Division, Brigham and Women's Hospital, 75 Francis Street, Boston, MA 02115.

Received for publication 26 September 1991 and in revised form 9 April 1992.

1. Abbreviations used in this paper: EDRF, endothelium-derived relaxing factor.

J. Clin. Invest.

(C) The American Society for Clinical Investigation, Inc.

0021-9738/92/10/1248/06 \$2.00

Volume 90, October 1992, 1248-1253 which is capable of inducing vasodilation and inhibiting platelet aggregation $(2,3)$. EDRF has been shown to be nitric oxide or a labile nitroso compound that liberates nitric oxide (4-6). As is the case with other nitrovasodilators, EDRF causes vasodilation by stimulating the activity of soluble guanylate cyclase within the vascular smooth muscle, thereby elevating tissue levels of cyclic GMP (7). EDRF is derived from the terminal guanidino nitrogen of $\mathrm{L}$-arginine (7-9). The exact mechanism whereby L-arginine is metabolized to nitric oxide in the endothelium is not known, but a soluble enzyme(s) requiring $\mathrm{NADPH}$, calcium, and calmodulin as cofactors is involved $(10,11)$.

A substantial amount of experimental evidence exists indicating that hyperlipoproteinemia impairs endothelium-dependent vasorelaxation, even before the development of atherosclerosis (12-23). We hypothesized that administration of $L$-arginine may increase synthesis of EDRF and thereby improve endothelium-dependent vasodilation in hypercholesterolemia. Indeed, our earlier studies conducted in cholesterol-fed rabbits support this contention $(20,24,25)$. In this report, we present data that extend these observations and demonstrate that L-arginine augments endothelium-dependent vasodilation in forearm resistance vessels of hypercholesterolemic humans.

\section{Methods}

Subjects. The control subject population in this study included 11 normal volunteers comprising 10 males and 1 female. Their ages ranged from 31 to 49 and averaged $39 \pm 2 \mathrm{yr}$. Normalcy was determined by a careful history, physical examination, and laboratory analysis to exclude individuals with hematologic, renal, or hepatic dysfunction. There were 14 patients with hypercholesterolemia. Hypercholesterolemia was defined as a serum LDL cholesterol level greater than the 75 th percentile adjusted for age and sex. These individuals included 11 males and 3 females whose ages ranged from 22 to 48 and averaged $38 \pm 2 \mathrm{yr}$. The ages of the normal volunteers and patients with hypercholesterolemia were not significantly different. None of the patients had historical evidence of atherosclerosis as determined by the absence of symptoms of angina, claudication, or cerebrovascular ischemia, nor clinical evidence of arterial occlusive disease as would be suggested by decreased pulses, asymmetric blood pressure, or bruits. In addition, no patient had hypertension, diabetes mellitus, or congestive heart failure. None of the subjects were taking diuretics, vasoactive medications, or nonsteroidal antiinflammatory medications. This study was approved by the Human Research Committee of Brigham and Women's Hospital and each subject gave written informed consent.

Experimental protocol. Each subject was studied in a $23^{\circ} \mathrm{C}$ temperature-controlled room in the postabsorptive state. Alcohol, caffeine, and cigarettes were all prohibited within $12 \mathrm{~h}$ of the study. Under local anesthesia and sterile conditions, a polyethylene catheter was inserted into a brachial artery of each subject for determination of blood pressure and for infusion of drugs. A separate polyethylene catheter was inserted into the antecubital vein for infusion of $\mathrm{L}$-arginine. The vascular research laboratory was quiet and lights were dimmed. All subjects rested at least $30 \mathrm{~min}$ after catheter placement to establish a stable baseline before data collection. 
During the control period, measurements of forearm blood flow and blood pressure were repeated every $10 \mathrm{~min}$ until stable. Dextrose $5 \%$ was infused intraarterially at a rate of $0.4 \mathrm{ml} / \mathrm{min}$ during the control period. To determine the maximal vasodilative potential of the resistance vessels, forearm blood flow was measured in the basal state and during reactive hyperemia after $5 \mathrm{~min}$ of an ischemic stimulus. Ischemia was induced by inflation of a sphygmomanometric cuff on the upper arm to suprasystolic pressure. Abnormalities in reactive hyperemic blood flow often imply structural problems in the resistance vessels preventing maximal vasodilation. Forearm blood flow measurements were repeated until basal conditions were reestablished.

The protocol used to examine forearm vascular reactivity in normal subjects and patients with hypercholesterolemia had been reported previously (22). To assess specifically endothelium-dependent vasodilation, methacholine chloride (a congener of acetylcholine) was administered via the brachial artery. Forearm blood flow was measured during infusion of methacholine chloride at concentrations of $0.3,3$, and $10 \mu \mathrm{g} / \mathrm{min}$ each for $3 \mathrm{~min}$, delivered at a rate of $0.4 \mathrm{ml} / \mathrm{min}$. To distinguish abnormalities in endothelial function from abnormalities of vascular smooth muscle, subjects received an intraarterial infusion of sodium nitroprusside. This agent, which acts directly on vascular smooth muscle by stimulating soluble guanylate cyclase and inducing hyperpolarization, was given at doses of $0.3,3$, and $10 \mu \mathrm{g} / \mathrm{min}$ at a rate of $0.4 \mathrm{ml} / \mathrm{min}$, each for 3-min period. The order of administration for methacholine chloride and sodium nitroprusside was randomized for each subject. Basal conditions were reestablished before each intervention. The doses of each drug were chosen to achieve decreases in forearm vascular resistance without causing systemic effects. Dose-response curves were generated for each drug infusion.

After completion of the methacholine chloride and sodium nitroprusside infusions, all normal subjects and 10 individuals with hypercholesterolemia were given L-arginine intravenously at a rate of 10 $\mathrm{mg} / \mathrm{kg}$ per min. This dose had been used in our previous study in rabbits (20). L-arginine was administered for a 20-min period to evaluate the effects of $\mathrm{L}$-arginine alone on forearm blood flow and vascular resistance. The infusion of L-arginine was then continued during repeated intraarterial infusions of methacholine chloride and sodium nitroprusside. The order of drug administration again was randomized.

D-arginine, the enantiomer of $\mathrm{L}$-arginine, is not a precursor of EDRF. Thus, to ensure that any observed effects of $L$-arginine were due to its contribution to the synthesis of EDRF and not just secondary to its physiochemical properties, five individuals with hypercholesterolemia received D-arginine, $10 \mathrm{mg} / \mathrm{kg}$ per min intravenously in a protocol identical to that described for L-arginine. One of these subjects had received L-arginine on a separate day.

Hemodynamic measurements. Bilateral forearm blood flow was determined by venous occlusion strain gauge plethysmography, using calibrated mercury-in-silastic strain gauges, and expressed as $\mathrm{ml} / 100$ $\mathrm{ml}$ tissue per min (D. E. Hokanson Inc., Issaquah, WA) (26). Each arm was supported above heart level. Venous occlusion pressure averaged $35 \pm 5 \mathrm{mmHg}$. Circulation to the hand was prevented by inflating a wrist cuff to suprasystolic pressure before each forearm blood flow determination. Determination of forearm blood flow comprised at least five separate measurements performed at 10-15-s intervals. By measuring blood flow in the infused arm one can determine the direct effect of the vasoactive drug. By measuring blood flow in the noninfused arm, one can be assured that systemic effects have not occurred if no change in blood flow developed during the drug infusion. Forearm vascular resistance was calculated as the ratio of mean blood pressure to forearm blood flow and expressed as units reflecting $\mathrm{mmHg}$ per $\mathrm{ml} / 100 \mathrm{ml}$ tissue per min.

Blood pressure was measured via an intraarterial cannula which was attached to a pressure transducer (P23; Statham, Gould, Inc., Oxnard, CA) aligned to an amplifier on a physiologic recorder (Gould Inc.). Heart rate was determined from a simultaneously obtained electrocardiographic signal and calculated from the R-R interval.

Arginine and insulin assays. Venous blood was withdrawn during the baseline period and at completion of the arginine infusion for deter- mination of plasma arginine and insulin concentrations. Arginine increases secretion of insulin, a hormone that has intrinsic vasoactive effects $(27,28)$. Plasma arginine concentration was measured with an amino acid analyzer (model 7300; Beckman Instruments, Inc., Palo Alto, CA). Insulin concentration was determined by radioimmunoassay (Coat-A-Count Kit; Diagnostic Product Corp., Los Angeles, CA).

Statistical analysis. Results are presented as means \pm SE. Analysis of variance for independent groups was employed to compare the responses to the drug infusions between the normal and hypercholesterolemic groups. Single factor repeated measures analysis of variance followed by a Newman-Keuls post-hoc test was used to evaluate the effect of each drug within a group. Student's $t$ test was used to compare paired data only. For data determined to have a nonparametric distribution, the Wilcoxon Rank Sum and Signed Rank tests were used to analyze results between and within groups, respectively. Statistical significance was accepted at the $95 \%$ confidence interval, $P<0.05$.

\section{Results}

The pertinent biochemical and hemodynamic characteristics of the two groups of subjects are provided in Tables I and II. As defined by enrollment criteria, the plasma total cholesterol and LDL cholesterol were significantly higher in the hypercholesterolemic patients than in the normal subjects. There was no significant difference in the plasma HDL cholesterol, VLDL cholesterol, or triglyceride levels between the two groups of subjects. Baseline blood pressure, heart rate, forearm blood flow, and forearm vascular resistance did not differ between normal and hypercholesterolemic subjects. Furthermore, there was no significant difference in reactive hyperemic forearm blood flow or minimal forearm vascular resistance between the two groups.

Vasodilative responses to methacholine and nitroprusside. Intraarterial infusion of methacholine chloride caused a dosedependent and significant increase in forearm blood flow and decrease in forearm vascular resistance in both normal and hypercholesterolemic subjects. In the hypercholesterolemic subjects, however, cholinergic vasodilation was attenuated compared to normal subjects $(P<0.05)$ (Fig. 1$)$. The differences between the groups were significant at doses exceeding $0.3 \mu \mathrm{g} / \mathrm{min}$. The maximal forearm blood flow response to methacholine in normal subjects was $19.0 \pm 1.9 \mathrm{ml} / 100 \mathrm{ml}$ of tissue per min, and in hypercholesterolemic subjects, it was $13.7 \pm 1.7 \mathrm{ml} / 100 \mathrm{ml}$ of tissue per $\min (P<0.05)$. Similarly, forearm vascular resistance was significantly greater in the hypercholesterolemic subjects than in the normal subjects at the 3 $\mu \mathrm{g} / \mathrm{min}(13.9 \pm 3.4$ vs $6.8 \pm 0.8 \mathrm{U}, P<0.05)$ and $10 \mu \mathrm{g} / \mathrm{min}$ doses $(8.3 \pm 2.0$ vs $4.9 \pm 0.7 \mathrm{U}, P<0.05)$. No changes in forearm

Table 1. Lipid Profile in Normal and

Hypercholesterolemic Subjects

\begin{tabular}{lll}
\hline & \multicolumn{1}{c}{ Normal } & \multicolumn{1}{c}{ Hypercholesterolemic } \\
\hline Total cholesterol & $4.22 \pm 0.16(163 \pm 6)$ & $6.39 \pm 0.36(247 \pm 14)^{*}$ \\
LDL cholesterol & $2.76 \pm 0.10(107 \pm 4)$ & $4.65 \pm 0.36(180 \pm 14)^{*}$ \\
HDL cholesterol & $0.96 \pm 0.08(37 \pm 3)$ & $0.93 \pm 0.05(36 \pm 2)$ \\
VLDL cholesterol & $0.65 \pm 0.10(25 \pm 4)$ & $1.01 \pm 0.21(39 \pm 8)$ \\
Triglyceride & $1.41 \pm 0.21(125 \pm 19)$ & $2.21 \pm 0.45(196 \pm 40)$
\end{tabular}

Values in millimoles per liter, (milligrams per deciliter) are expressed as mean \pm SE. ${ }^{*} P<0.05$ vs. normal subjects. 
Table 2. Baseline Characteristics of Normal and Hypercholesterolemic Subjects

\begin{tabular}{llc}
\hline & Normal & Hypercholesterolemic \\
\hline Heart rate (beats/min) & $57 \pm 3$ & $61 \pm 2$ \\
$\begin{array}{l}\text { Mean arterial pressure (mmHg) } \\
\text { Basal forearm blood flow }\end{array}$ & $84 \pm 2$ & $87 \pm 3$ \\
$\quad(\mathrm{ml} / 100 \mathrm{ml}$ tissue per min) & $3.0 \pm 0.5$ & $2.3 \pm 0.3$ \\
$\begin{array}{l}\text { Basal forearm vascular resistance (units) } \\
\text { Reactive hyperemic forearm blood flow }\end{array}$ & $36 \pm 6$ & $43 \pm 5$ \\
$\quad$ (ml/100 ml tissue per min) & $29 \pm 2$ & $27 \pm 2$ \\
$\begin{array}{l}\text { Minimal forearm vascular resistance } \\
\quad \text { (units) }\end{array}$ & $3.0 \pm 0.3$ & $3.3 \pm 0.3$ \\
\hline
\end{tabular}

Values are expressed as mean \pm SEM. There was no significant difference between groups for any hemodynamic variable.

blood flow occurred in the noninfused arm in either subject group. In addition, intraarterial infusion of methacholine chloride caused no changes in blood pressure or heart rate in either group of subjects.

Infusion of sodium nitroprusside also increased forearm blood flow and decreased forearm vascular resistance in normal and hypercholesterolemic subjects. In contrast to the attenuated response to methacholine chloride observed in the hypercholesterolemic patients, the effects of sodium nitroprusside on forearm blood flow and forearm vascular resistance did not differ significantly between normal and hypercholesterolemic subjects (Fig. 2). At a dose of $10 \mu \mathrm{g} / \mathrm{min}$, forearm blood flow was $12.8 \pm 1.3$ and $10.3 \pm 1.0 \mathrm{ml} / 100 \mathrm{ml}$ of tissue per $\mathrm{min}$, and forearm vascular resistance was $7.0 \pm 0.8$ and $9.2 \pm 1.1 \mathrm{U}$ in normal and hypercholesterolemic subjects, respectively (each $P=$ NS). No changes in forearm blood flow or forearm vascular resistance occurred in the noninfused arm of either group of subjects. Taken together, these data demonstrate that endothe-

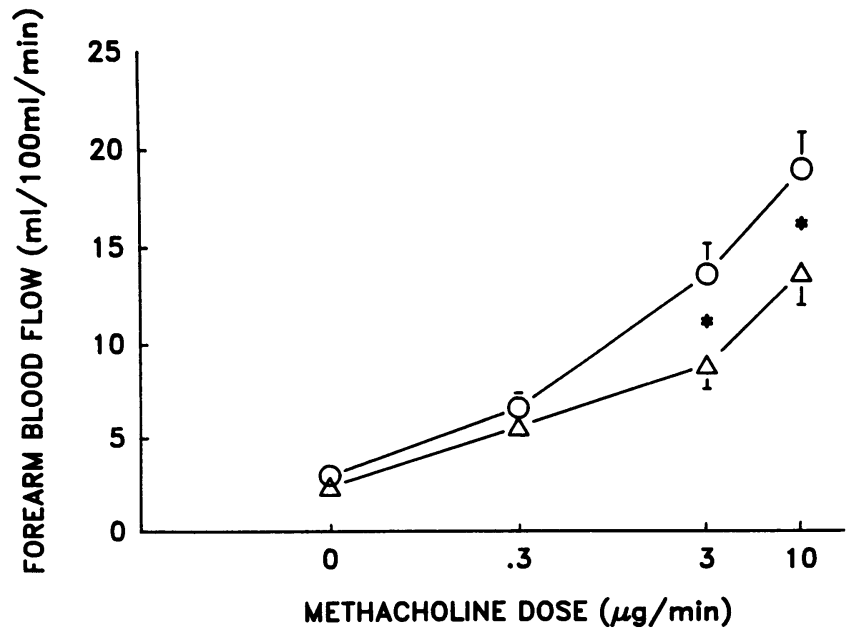

Figure 1. The forearm blood flow responses to intraarterial infusion of methacholine chloride in normal $(n=11)$ and hypercholesterolemic ( $n=14)$ subjects. Cholinergic vasodilation was less in the hypercholesterolemic group than in the normal group. The difference between groups was significant at the 3 and $10 \mu \mathrm{g} / \mathrm{min}$ doses. $\Delta$, Hypercholesterolemic; $\circ$, normal; ${ }^{*} P<0.05$.

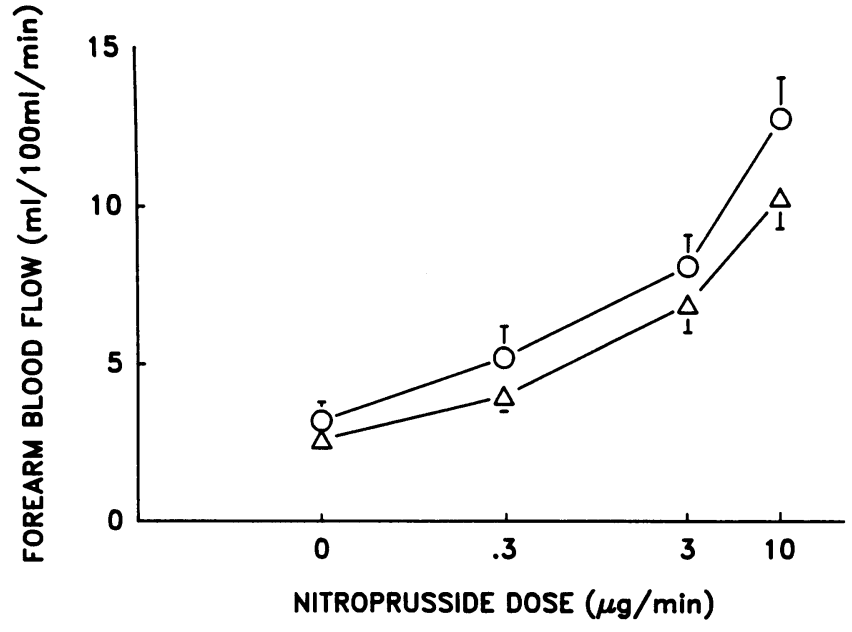

Figure 2. The forearm blood flow response to intraarterial infusion of sodium nitroprusside in normal $(n=11)$ and hypercholesterolemic ( $n=14)$ subjects. There was no significant difference in the dose-response relationship between the two groups. $\Delta$, Hypercholesterolemic; o, normal.

lium-dependent vasodilation is impaired in patients with hypercholesterolemia.

Effect of L-arginine on endothelium-dependent vasodilation. L-arginine, when infused alone, did not affect blood pressure, heart rate, forearm blood flow, or forearm vascular resistance in either group of subjects. In the normal subjects, L-arginine did not potentiate the vasodilation that occurred during the administration of methacholine chloride (Fig. 3). In the hypercholesterolemic subjects, however, the L-arginine infusion augmented the vasodilative response to methacholine chloride. The increase in forearm blood flow was greater at the $3(P$ $<0.05)$ and $10 \mu \mathrm{g} / \mathrm{min}$ doses $(P<0.01)$ (Fig. 4$)$. L-arginine did not potentiate the vasodilation caused by sodium nitroprusside in either normal or hypercholesterolemic subjects.

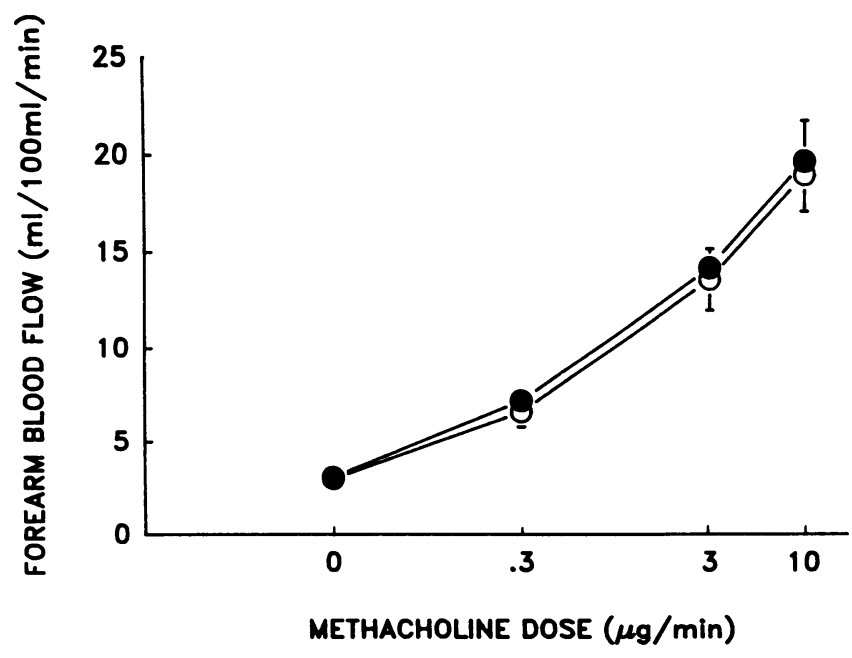

Figure 3. The effect of intravenous L-arginine on the forearm blood flow response to intraarterial methacholine chloride in normal subjects $(n=11)$. L-arginine did not potentiate the vasodilation that occurred during the administration of methacholine chloride. $\circ$, Pre-L-arginine; $\bullet$, post-L-arginine. 
Effect of D-arginine on vasodilation in hypercholesterolemic subjects. Basal values for blood pressure, heart rate, forearm blood flow, and forearm vascular resistance were not significantly different between the hypercholesterolemic patients who received $\mathrm{D}$-arginine and those who received L-arginine. Intravenous infusion of D-arginine had no effect on any of these hemodynamic measurements. The forearm vascular responses to methacholine (Fig. 5) and sodium nitroprusside were not altered significantly by D-arginine.

Plasma arginine and insulin concentrations. Intravenous infusion of L-arginine increased the plasma concentration of arginine from $102 \pm 5$ to $8,172 \pm 122 \mu \mathrm{M} /$ liter in the normal subjects $(n=4)$ and from $106 \pm 9$ to $7,157 \pm 472 \mu \mathrm{M} /$ liter in the hypercholesterolemic subjects $(n=3)$. D-arginine increased plasma arginine concentration from $84 \pm 29$ to $9,992 \pm 294 \mu \mathrm{M} /$ liter in the hypercholesterolemic subjects $(n=2)$. During the infusion of L-arginine, serum insulin concentration increased from $9 \pm 1$ to $38 \pm 2 \mu \mathrm{U} / \mathrm{ml}$ in normal subjects $(n=4)$, and from $12 \pm 1$ to $48 \pm 15 \mu \mathrm{U} / \mathrm{ml}$ in the hypercholesterolemic subjects $(n=3)$. When D-arginine was administered to hypercholesterolemic individuals, serum insulin levels increased from $18 \pm 4$ to $47 \pm 13 \mu \mathrm{U} / \mathrm{ml}(n=5)$. The magnitude of increase in serum insulin concentration was comparable during both $\mathrm{L}$-arginine and D-arginine infusions.

\section{Discussion}

The important findings in this study are: $(a)$ endothelium-dependent vasodilation is abnormal in forearm resistance vessels of hypercholesterolemic humans; and $(b)$ intravenous administration of L-arginine improves endothelium-dependent vasodilation in these individuals. This discussion will provide additional evidence that hypercholesterolemia impairs endothelium-dependent vasodilation, examine the role of $\mathrm{L}$-arginine in the synthesis of EDRF, review data from previous studies in animals that support our observations in humans, and consider the clinical implications of our findings.

Abnormal endothelium-dependent vasorelaxation in hypercholesterolemia. A cholesterol-enriched diet is often used to induce atherosclerosis in certain species. Aortae isolated from

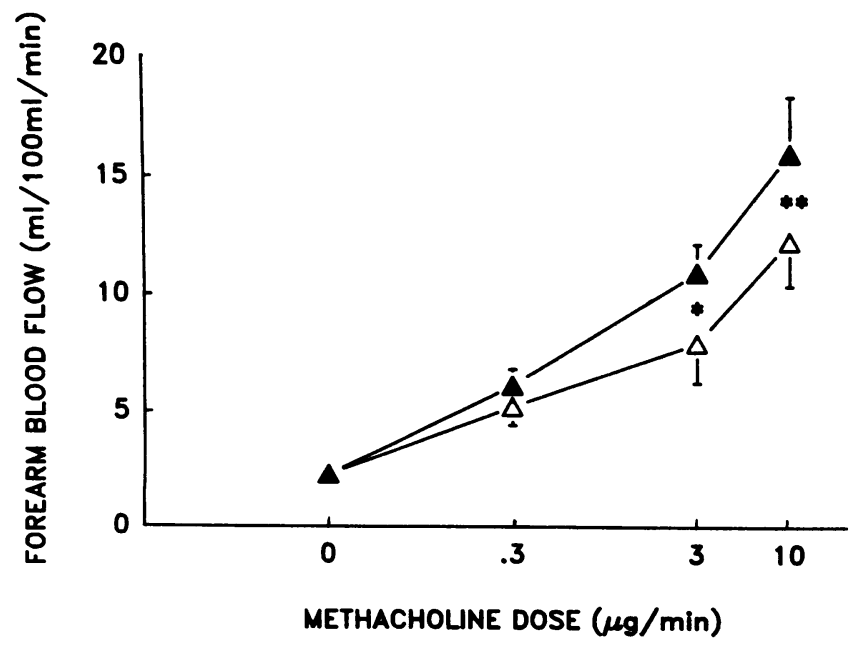

Figure 4. The effect of intravenous L-arginine on the forearm blood flow response to intraarterial methacholine chloride in hypercholesterolemic subjects $(n=10)$. L-arginine augmented cholinergic vasodilation in this group. $\triangle$, Pre-L-arginine; $\triangle$, post-L-arginine; ${ }^{*} P<0.05$; ${ }^{* *} P<0.01$.

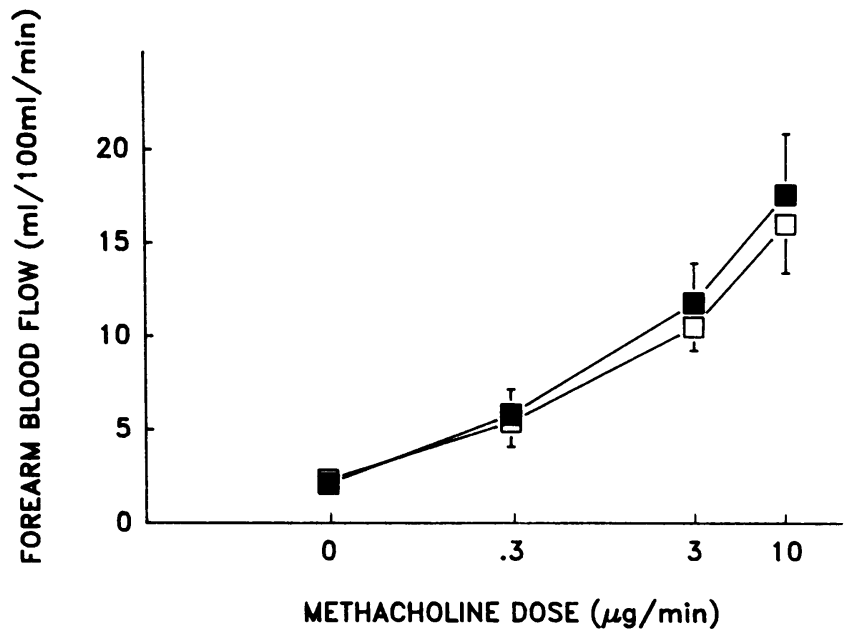

Figure 5. The effect of intravenous D-arginine on the forearm blood flow response to intraarterial methacholine chloride in hypercholesterolemic subjects $(n=5)$. D-arginine did not potentiate cholinergic vasodilation. $\square$, Pre-D-arginine; $\square$, post-D-arginine.

cholesterol-fed rabbits with atherosclerosis relax less to the endothelium-dependent vasodilator acetylcholine, than aortae from control rabbits, whereas endothelium-independent vasodilation is generally preserved $(29,30)$. Similarly, endothelium-dependent vasorelaxant responses to acetylcholine and thrombin are attenuated in iliac arteries excised from cynomolgus monkeys with diet-induced atherosclerosis (31). Likewise, atherosclerotic human coronary arteries respond abnormally to intracoronary infusion of acetylcholine, but not nitroglycerin $(32,33)$. Multivariate analysis suggests that hypercholesterolemia is one of the most important factors predicting abnormal endothelial function in these vessels (34). It may be inferred from these studies that hypercholesterolemia impairs endothelial function; however, interpretation is confounded by the presence of morphologic changes due to atherosclerosis.

To circumvent this problem, several groups of investigators have examined the direct effect of lipoproteins in vitro and reported that incubation of normal vascular strips with LDL cholesterol inhibit endothelium-dependent vasorelaxation (12-14). Chronic in vivo exposure to high levels of cholesterol also inhibit endothelium-dependent relaxation of excised porcine coronary arteries, even when there is no histologic evidence of atherosclerosis $(15,16)$. Furthermore, endotheliumdependent vasodilation is abnormal in hind limb, cremasteric, and coronary resistance vessels of cholesterol-fed rabbits (1721 ). We have reported previously that vasodilative function is impaired in the forearm resistance vessels of hypercholesterolemic humans (22). Unlike the larger arteries, the walls of resistance vessels do not develop atheroma $(18,21)$. Thus, hypercholesterolemia can adversely affect endothelial function in the absence of atherosclerosis.

The precise mechanism by which hypercholesterolemia impairs endothelium-dependent vasodilation is not known. Potential explanations for this phenomenon include reduced synthesis or release of EDRF, increased degradation of EDRF, and competing vasoconstrictive stimuli (35-38).

L-arginine augments endothelium-dependent vasodilation. If hypercholesterolemia reduces the synthesis and/or release of EDRF, or otherwise limits its availability, endothelium-dependent vascular responses possibly may be restored by supplying the precursor of EDRF, L-arginine. Normally, L-arginine does 
not affect endothelium-dependent vasorelaxation, indicating that intracellular stores of $L$-arginine are sufficient to saturate the enzyme responsible for EDRF synthesis (20, 39-42). Under certain conditions, however, exogenous L-arginine is required to normalize endothelium-dependent relaxation. For example, bovine aortic endothelial cells cultured in medium deficient in $\mathrm{L}$-arginine release little EDRF, as detected by bioassay and chemiluminescence studies. When L-arginine is added to these cells, increased amounts of EDRF are released (43). Also, endothelium-dependent vasorelaxation is markedly reduced in isolated vessels after either prolonged tension or exposure to calcium ionophore, presumably due to overutilization of intracellular L-arginine stores (44-46). Addition of L-arginine restores endothelium-dependent relaxation. Antagonists of EDRF synthesis, such as $N^{\mathrm{G}}$-monomethyl-L-arginine, attenuate endothelium-dependent relaxation in vitro and in vivo $(40-42,47)$. Normal responses return after the administration of L-arginine. For these reasons, we have hypothesized that administration of exogenous L-arginine may correct impaired endothelium dependent vasodilation caused by hypercholesterolemia.

In this regard, we performed a series of experiments in cholesterol-fed rabbits. In one study, endothelium-dependent and independent vasodilation of perfused basilar arteries was examined in vitro (24). After incubation of the vessels with L-arginine, endothelium-dependent vasodilation was normalized in the vessels harvested from the hypercholesterolemic animals. In a second set of experiments performed in vivo, intravenous administration of $\mathbf{L}$-arginine improved endothelium-dependent vasodilation in the hind limb resistance vessels of cholesterol-fed, but not control, rabbits (20). L-arginine did not affect endothelium-independent vasodilation in either group of rabbits. In a third study, we found that endothelium-dependent vasodilation was preserved in aortae excised from cholesterolfed rabbits who received L-arginine in vivo, but was blunted in those rabbits who received vehicle (25).

The current study extends these observations to humans. L-arginine augmented endothelium-dependent vasodilation induced by methacholine in the hypercholesterolemic group but not in the normal subjects. An effect of L-arginine on endothelium-independent vasodilation can be excluded since the vasodilative response to sodium nitroprusside was not potentiated. The fact that D-arginine had no effect on endotheliumdependent vasodilation is consistent with our understanding that L-arginine is a specific substrate for the synthesis of nitric oxide in vivo.

Several circumstances may explain why L-arginine augments endothelium-dependent vasodilation in the hypercholesterolemic subjects. Hypercholesterolemia may cause intracellular depletion of $\mathrm{L}$-arginine by reducing transmembrane transport. Alternatively, increased degradation of EDRF may drive the synthetic pathway until arginine stores are depleted. Finally, there may be reduced substrate affinity for the enzyme, EDRF synthase. Our results demonstrate that L-arginine restores endothelial function acutely in hypercholesterolemic subjects, but does not specifically address which of these mechanisms may be responsible for the abnormal vasodilator function in these individuals.

It is possible that $\mathrm{L}$-arginine increased forearm blood flow by a mechanism unrelated to nitric oxide synthesis. For example, $\mathrm{L}$-arginine increases insulin release from pancreatic islet cells (27). An increase in serum insulin levels might potentially increase forearm blood flow, as limb vasodilation has been shown to occur during intraarterial insulin infusion in man (28). In this study, however, serum insulin concentration increased similarly during the infusions of D-arginine and $\mathrm{L}$-arginine, yet only $\mathrm{L}$-arginine potentiated the blood flow response to methacholine, and only in the hypercholesterolemic subjects. Thus, it is unlikely that increased insulin release can explain the potentiated vasodilator response to L-arginine in hypercholesterolemic subjects.

It is also unlikely that vasodilator prostaglandins contributed to the observed forearm hemodynamic responses to methacholine. We and others have previously examined the forearm vasodilative response to methacholine or acetylcholine before and after administration of either aspirin or indomethacin (22, $48,49)$. These cyclooxygenase inhibitors did not affect cholinergic-induced vasodilation.

Clinical implications. This study confirms previous observations in animals and humans that hypercholesterolemia impairs endothelium-dependent vasodilation. The principal new observation is that this abnormality can be improved acutely by administration of $L$-arginine. The major implication of our results is that the defect is reversible. Thus, it may be possible to develop long-term strategies to correct abnormal endothelial function caused by hypercholesterolemia. L-arginine is a semiessential amino acid that is found in high concentrations in fish, poultry, and beans. It is conceivable that a diet enriched with L-arginine may prevent abnormal endothelial function in patients with hypercholesterolemia. In animals, altering the lipid milieu by administration of fish oil, or reducing high cholesterol levels by diet or administration of a hepatic hydroxymethyl glutaryl CoA reductase inhibitor has been shown to improve endothelium dependent vasodilation $(31,50,51)$. Whether these interventions will be effective in humans is not yet established.

EDRF not only causes vasodilation, but it also inhibits platelet aggregation and suppresses vascular smooth muscle proliferation. Hence, in the presence of hypercholesterolemia, impaired synthesis or release of EDRF may promote abnormal platelet-vascular wall interaction, possibly facilitating the uptake and activation of circulating macrophages as well as the expression of endogenous mitogens, and thereby contributing to the development of atherosclerosis (52). It is intriguing to speculate that chronic dietary or therapeutic interventions can be instituted in hypercholesterolemic humans that will preserve endothelial function and obviate the pathologic consequences of hypercholesterolemia.

\section{Acknowledgments}

This study was supported by a Grant-in-Aid award from the American Heart Association and Bristol-Myers. Dr. Creager is a recipient of a National Institutes of Health Research Career Development Award, HL-01768.

\section{References}

1. Vane, J. R., E. E. Änggard, and R. M. Botting. 1990. Regulatory functions of the vascular endothelium. N. Engl. J. Med. 323:27-36.

2. Furchgott, R. F., and J. V. Zawadzki. 1980. The obligatory role of endothelial cells in the relaxation of arterial smooth muscle by acetylcholine. Nature (Lond.). 288:373-376.

3. Shepherd, J. T., and P. M. Vanhoutte. 1991. Endothelium-derived relaxing (EDRF) and contracting factors (EDCF) in the control of cardiovascular homeostasis: the pioneering observations. In Cardiovascular Significance of Endothelium-Derived Vasoactive Factors. G. M. Rubanyi, editor. Futura Publishing Co., Mt. Kisco, NY. 39-64.

4. Palmer, R. M. J., A. G. Ferridge, and S. Moncada. 1987. Nitric oxide 
release accounts for the biological activity of endothelium-derived relaxing factor. Nature (Lond.). 327:524-526.

5. Ignarro, L. J., R. E. Byrns, G. M. Buga, and K. S. Wood. 1987. Endothelium-derived relaxing factor from pulmonary artery and vein possesses pharmacologic and chemical properties identical to those of nitric oxide radical. Circ. Res. 61:866-879.

6. Myers, P. R., R. Guerra, and D. G. Harrison. 1989. Release of NO and EDRF from cultured bovine aortic endothelial cells. Am. J. Physiol. 256 (Heart Circ. Physiol. 25):H1030-H1037.

7. Ignarro, L. J. 1989. Biological actions and properties of endothelium-derived nitric oxide formed and released from artery and vein. Circ. Res. 65:1-21.

8. Palmer, R. M. J., D. S. Ashton, and S. Moncada. 1988. Vascular endothelial cells synthesize nitric oxide from L-arginine. Nature (Lond.). 333:664-666.

9. Sakuma, I., D. Stuehr, S. S. Gross, C. Nathan, and R. Levi. Identification of arginine as a precursor of endothelium-derived relaxing factor. 1988. Proc. Natl. Acad. Sci. USA. 85:8664-8667.

10. Palmer, R. M. J., and S. Moncada. 1989. A novel citrulline-forming enzyme implicated in the formation of nitric oxide by vascular endothelial cells. Biochem. Biophys. Res. Commun. 158:348-352.

11. Marletta, M. A. 1989. Nitric oxide: biosynthesis and biological signifcance. Trends Biochem. Sci. 14:488-492.

12. Andrews, H. E., K. R. Bruckdorfer, R. C. Dunn, and M. Jacobs. Low-density lipoproteins inhibit endothelium-dependent relaxation in rabbit aorta. 1987. Nature (Lond.). 327:237-239.

13. Tomita, T., M. Ezaki, M. Miwa, K. Nakamura, and Y. Inoue. 1990. Rapid and reversible inhibition by low density lipoprotein of the endothelium-dependent relaxation to hemostatic substances in porcine coronary arteries. Circ. Res. 66:18-27.

14. Takahashi, M., Y. Yui, H. Yasumoto, T. Aoyama, H. Morishita, R. Hattori, and C. Kiawai. Lipoproteins are inhibitors of endothelium-dependent relaxation of rabbit aorta. 1990. Am. J. Physiol. 258 (Heart Circ. Physiol. 27):H1-H8.

15. Cohen, R. A., K. M. Zitnay, C. C. Haudenschild, and L. D. Cunningham. 1988. Loss of selective endothelial cell vasoactive functions in pig coronary arteries caused by hypercholesterolemia. Circ. Res. 63:903-910.

16. Shimokawa, H., and P. M. Vanhoutte. 1989. Impaired endothelium-dependent relaxation to aggregating platelets and related vasoactive substances in porcine coronary arteries in hypercholesterolemia and atherosclerosis. Circ. Res. 64:900-914.

17. Bossaller, C., H. Yamamoto, P. R. Lichtlen, and P. D. Henry. 1987. Impaired cholinergic vasodilation in the cholesterol-fed rabbit in vivo. Basic Res. Cardiol. 82:396-404.

18. Osborne, J. A., M. J. Siegman, A. W. Sedar, S. U. Mooers, and A. M. Lefer. 1989. Lack of endothelium-dependent relaxation in coronary resistance arteries of cholesterol-fed rabbits. Am. J. Physiol. 256:C591-C597.

19. Wright, C. E., and J. A. Angus. 1986. Effects of hypertension and hypercholesterolemia on vasodilation in the rabbit. Hypertension (Dallas). 8:361-371.

20. Girerd, X. J., A. T. Hirsch, J. P. Cooke, and M. A. Creager. 1990. L-arginine augments endothelium-dependent vasodilation in cholesterol-fed rabbits. Circ. Res. 67:1301-1308.

21. Yamamato, H., C. Bossaller, J. Cartwright, Jr., and P. D. Henry. 1988. Videomicroscopic demonstration of defective cholinergic arteriolar vasodilation in atherosclerotic rabbit. J. Clin. Invest. 81:1752-1758.

22. Creager, M. A., J. P. Cooke, M. D. Mendelsohn, S. G. Gallagher, S. M. Coleman, J. Loscalzo, and V. J. Dzau. 1990. Impaired vasodilation of forearm resistance vessels in hypercholesterolemic humans. J. Clin. Invest. 86:228-234.

23. Zeiher, A. M., H. Drexler, H. Wollschläger, and J. Hanjörg. 1991. Modulation of coronary vasomotor tone in humans. Circulation. 83:391-401.

24. Rossitch, E., Jr., E. Alexander III, P. M. Black, and J. P. Cooke. 1991. L-arginine normalizes endothelial function in cerebral vessels from hypercholesterolemic rabbits. J. Clin. Invest. 87:1295-1299.

25. Cooke, J. P., N. A. Andon, X. J. Girerd, A. T. Hirsch, and M. A. Creager 1991. Arginine restores cholinergic relaxation of hypercholesterolemic rabbit thoracic aorta. Circulation. 83:1057-1062.

26. Hokanson, D. E., D. S. Sumner, and D. E. Strandness, Jr. 1975. An electrically calibrated plethysmograph for direct measurement of limb blood flow. IEEE (Inst. Electr. Electron. Eng.) Trans. Biomed. Eng. 22:25-29.

27. Floyd, J. C., S. S. Fajans, J. W. Conn, R. F. Knopf, and J. Rull. 1966 Stimulation of insulin secretion by amino acids. J. Clin. Invest. 9:1487-1502.

28. Creager, M. A., C.-S. Liang, and J. D. Coffman. 1985. Beta adrenergic-mediated vasodilator response to insulin in the human forearm. J. Pharmacol. Exp. Ther. 235:709-714.

29. Verbeuren, T. J., F. H. Jordaens, L. L. Zonnekeyn, C. E. Van Hove, M.-C Coene, and A. G. Herman. 1986. Effect of hypercholesterolemia on vascular reactivity in the rabbit. I. Endothelium-dependent and endothelium-independent contractions and relaxations in isolated arteries of control and hypercholesterolemic rabbits. Circ. Res. 58:552-564.

30. Habib, J. B., C. Bossaller, S. Wells, C. Williams, J. D. Morrisett, and P. D. Henry. 1986. Preservation of endothelium-dependent vascular relaxation in cholesterol-fed rabbit by treatment with the calcium blocker PN 200110. Circ. Res. 58:305-309.

31. Harrison, D. G., M. L. Armstrong, P. C. Freiman, and D. D. Heistad. 1987. Restoration of endothelium-dependent relaxation by dietary treatment of atherosclerosis. J. Clin. Invest. 80:1808-1811.

32. Ginsberg, R., M. R. Bristow, K. Davies, A. Didiasc, and M. E. Billingham. 1984. Quantitative pharmacologic responses of normal and atherosclerotic isolated human epicardial coronary arteries. Circulation. 69:430-440.

33. Ludmer, P. L., A. P. Selwyn, T. L. Shook, R. R. Wayne, G. H. Mudge, R. W. Alexander, and P. Ganz. 1986. Paradoxical vasoconstriction induced by acetylcholine in atherosclerotic coronary arteries. N. Engl. J. Med. 315:10461051.

34. Vita, J. A., C. B. Treasure, E. G. Nabel, J. M. McLenachan, R. D. Fish, A. C. Yeung, V. I. Vekshtein, A. P. Selwyn, and P. Ganz. 1990. Coronary vasomotor response to acetylcholine relates to risk factors for coronary artery disease. Circulation. 81:491-497.

35. Tagawa, H., H. Tomoike, and M. Nakamura. 1991. Putative mechanisms of the impairment of endothelium-dependent relaxation of the aorta with atheromatous plaque in heritable hyperlipidemic rabbits. Circ. Res. 68:330-337.

36. Minor, R. L., Jr., P. R. Myers, R. Guerra, Jr., J. N. Bates, and D. G. Harrison. 1990. Diet-induced atherosclerosis increases the release of nitrogen oxides from rabbit aorta. J. Clin. Invest. 86:2109-2116.

37. Simon, B. C., L. D. Cunningham, and R. A. Cohen. 1990. Oxidized low density lipoproteins cause contraction and inhibit endothelium-dependent relaxation in the pig coronary artery. J. Clin. Invest. 86:75-79.

38. Galle, J., E. Bassenge, and R. Busse. 1990. Oxidized low density lipoproteins potentiate vasoconstriction to various agonists by direct interaction with vascular smooth muscle. Circ. Res. 66:1287-1293.

39. Thomas, G., R. Vargas, B. Wroblewska, and P. W. Ramwell. 1989. Role of the endothelium and arginine peptides on the vasomotor response of porcine internal mammary artery. Life Sci. 44:1823-1830.

40. Aisaka, K., S. S. Gross, O. W. Griffith, and R. Levi. 1989. NG-methylarginine, an inhibitor of endothelium-derived nitric oxide synthesis, is a potent pressor agent in the guinea pig: does nitric oxide regulate blood pressure in vivo? Biochem. Biophys. Res. Commun. 160:881-886.

41. Amezcua, J. L., R. M. J. Palmer, B. M. De Souza, and S. Moncada. 1989. Nitric oxide synthesized from L-arginine regulates vascular tone in the coronary circulation of the rabbit. Br. J. Pharmacol. 97:1119-1124.

42. Rees, D. D., R. M. J. Palmer, and S. Moncada. 1989. Role of endothelium-derived nitric oxide in the regulation of blood pressure. Proc. Natl. Acad. Sci. USA. 86:3375-3378.

43. Palmer, R. M., D. D. Rees, D. S. Ashton, and S. Moncada. 1988. L-arginine is the physiological precursor for the formation of nitric oxide in endothelium-dependent relaxation. Biochem. Biophys. Res. Commun. 153:1251-1256.

44. Gold, M. E., K. S. Wood, G. M. Buga, R. E. Byrns, and L. J. Ignarro. 1989. $\mathrm{L}$-arginine causes whereas $\mathrm{L}$-argininosuccinic acid inhibits endothelium-dependent vascular smooth muscle relaxation. Biochem. Biophys. Res. Commun. 161:536-543.

45. Gold, M. E., K. S. Wood, R. E. Byrns, G. M. Buga, and L. J. Ignarro. 1990. L-arginine-dependent vascular smooth muscle relaxation and cGMP formation. Am. J. Physiol. 259:H1813-H1821.

46. Schini, V. B., and P. M. Vanhoutte. 1991. Endothelium-dependent and -independent relaxations in L-arginine-depleted aortas of the rat. Circ. Res. 68:209-216.

47. Vallance, P., J. Collier, and S. Moncada. 1989. Effects of endotheliumderived nitric oxide on peripheral arteriolar tone in man. Lancet. ii:997-1000.

48. Linder, L., W. Kiowski, F. R. Bühler, and T. F. Lüscher. 1990. Indirect evidence for release of endothelium-derived relaxing factor in human forearm circulation in vivo. Circulation. 81:1762-1767.

49. Panza, J. A., A. A. Quyyumi, J. E. Brush, Jr., and S. E. Epstein. 1990. Abnormal endothelium-dependent vascular relaxation in patients with essential hypertension. N. Engl. J. Med. 323:22-27.

50. Shimokawa, H., and P. M. Vanhoutte. 1988. Dietary cod-liver oil improves endothelium-dependent responses in hypercholesterolemic and atherosclerotic porcine coronary arteries. Circulation. 78:1421-1430.

51. Osborne, J. A., P. H. Lento, M. R. Siegfried, G. L. Stahl, B. Fusman, and A. M. Lefer. 1989. Cardiovascular effects of acute hypercholesterolemia in rabbits. Reversal with lovastatin treatment. J. Clin. Invest. 83:465-473.

52. Ross, R. 1986. The pathogenesis of atherosclerosis: an update. $N$. Engl. J. Med. 314:488-500. 